\title{
Accuracy Comparison of the Optical 3D Scanner and CT Scanner
}

\author{
Radomir Mendricky ${ }^{1}$, Jiri Sobotka ${ }^{2}$ \\ ${ }^{1}$ Faculty of Mechanical Engineering, Department of Manufacturing Systems and Automation, Technical University \\ of Liberec. Studentska 1402/2, 46117 Liberec. Czech Republic. E-mail: radomir.mendricky@tul.cz \\ ${ }^{2}$ Faculty of Mechanical Engineering, Department of Engineering Technology, Technical University of Liberec. \\ Studentska 1402/2, 46117 Liberec. Czech Republic. E-mail: jiri.sobotka@tul.cz
}

\begin{abstract}
Regarding the very dynamic development of the non-contact measurement methods, there has been observed still increasing number of their applications in various fields during the last years - not only in the engineering industry. E.g. from the dimensional quality point of view, knowledge of real 3D data of a given part is truly very important. There are several options for obtaining these data such as the usage of optical 3D digitization or computed tomography (CT). However, within the mutual comparability of such data, it is very important to know not only the accuracy of acquiring $3 \mathrm{D}$ data, but also e.g. possibilities of these systems in terms of own measurement. In the paper, a specially designed part containing various convex and concave shapes was measured by using two different systems (ATOS TripleScan optical 3D scanner and METROTOM 1500 G2 CT scanner). The resulting scanned models were then compared not only in terms of dimensional accuracy, but also in terms of quality and detail of the obtained data or the time required to prepare the measurement and its implementation.
\end{abstract}

Keywords: 3D Digitalization, Computed Tomography, Optical 3D Scanning, CMM, Inspection

\section{Introduction}

Digitalization techniques (acquisition systems) represent one of the crucial approaches mainly in the field of quality control, inspection and e.g. reverse engineering [1]. In addition to that, so-called radiation method can be used both for the digitalization and for the non-destructive material testing. Regarding the great variety of these digitalization techniques and their rapid development over the last few years, there is always strong and desirable effort not only about precise classification, but also for their mutual comparison in light of accuracy, elimination of noise, hole filling, acquisition of the point cloud and so on. One of the first classification of the digitalization techniques was probably performed by Várady et al. [2] between contact-less methods (e.g. optical ones) and contact methods (e.g. CMMs - Coordinate Measuring Machines). In this article Várady gradually classified the optical methods into five categories as following triangulation, ranging, interferometry, structured lighting and image analysis. Moreover, quite a lot comparative studies were published to compare individual acquisitions systems from the different point of views - e.g. Bárbero and Ureta [3].

Also major aim of the submitted paper was to compare and evaluate two different acquisition systems (optical 3D scanner and computed tomography scanner) in two basic areas. The first of the assessed aspects was the accuracy of obtaining 3D data. In ad- dition to that there was also effort to summarize advantages and disadvantages of these two systems in terms of quality and comfort of scanning.

As there was already mentioned before, two different systems were used in the experimental part. The first of them was the optical 3D scanner ATOS III TripleScan from German company GOM Gmbh, which is used in various sectors of industry - e.g. construction, design, production, control quality, design and so on. As the second device there was used was a CT scanner METROTOM 1500 (2nd generation) from German company Carl Zeiss Industrielle Messtechnik $\mathrm{GmbH}$, designed for application of computed tomography (CT) to perform measuring and inspection jobs with only one $\mathrm{X}$-ray scan. This device is primarily designed for fast scan of large parts, which also means that its accuracy is a little bit lower than other products in this Zeiss METROTOM family.

The real dimensions (thus for purposes of this paper the nominal dimensions of the elements, which are hereinafter marked as the reference ones) were obtained from the measurement on the three-axis CMM (Coordinate Measuring Machine) DEA GLOBAL Status 7.10.5 from the manufacturer Hexagon Metrology Ltd. The CMM measurement accuracy was higher by more than order of magnitude than the estimated measurement accuracy both of optical 3D scanner ATOS III TripleScan and of CT scanner METROTOM 1500. The calibration list of the machine states the measurement accuracy of $2.5 \mu \mathrm{m}$. 
All of that was finally followed by a complete scanning of the chosen testing standard (model) both by optical 3D scanner ATOS TripleScan and CT scanner METROTOM 1500. To ensure high accuracy and reliability of scanned data, all measurements were performed under constant conditions - temperature $20 \pm$ $1{ }^{\circ} \mathrm{C}$ and relative humidity of $50 \pm 10 \%$. For their mutual comparison of scanned data and their comparison with data from CMM was used software GOM Inspect from the German company GOM Gmbh.

\section{Optical 3D scanner}

The professional system of the German company GOM, the ATOS III TripleScan System, was chosen as a representative of the optical 3D scanners. It is an optical 3D scanner in which the digitization process is based on the principles of so-called Fringe Projection, active triangulation and stereovision. In addition to that, the system uses LED projection of blue light (Blue Light Technology). By selecting suitable optical objectives, the device allows to define in the scanning direction the size of the 3D area, in which can be subsequently scanned the measured object - so-called measuring volume. This determines not only the size of the measured part, but also significantly affects the density of measured points and the own accuracy of scanning. In our case, the scanner was equipped with objectives having measuring volume of MV 320, which provides a theoretical density of measured points as $100 \mu \mathrm{m}$ under resolution of CCD cameras as 8 million pixels.

In the case of system ATOS III TripleScan, it was necessary to stick so-called reference points on the model before the actual scanning (due to the glossy surface) and to apply an anti-reflective spray of chalk powder. There was used 3-D Helling lase spraying spray. The thickness of the spray shouldn't significantly affect the accuracy of measured part, because acc. to our tests, the average spray thickness of this product varies around $0.013 \mathrm{~mm}$ [4]. This is also confirmed by Paloušek et al. [5], who focused his research on the effect of matt coatings on the accuracy of 3D optical measurements and found that while the chalk coating can reach a thickness up to $44 \mu \mathrm{m}$ on average, using anti-reflection coating based on titanium coating reduces the thickness by about ten times - so about 5 $\mu \mathrm{m}$. After such preparation, the measured part was subsequently fixed on a rotary measuring table and totally 14 scans were taken from each side under angle of $45^{\circ}$ - as it is shown in Fig. 1 - left. All data were transformed into a common global coordinate system using common reference points. Noise was erased and an optimized polygon mesh (format *.stl) was computed. This model was then used in accuracy analyses and for comparison with the model from the CT scanner. The entire measurement and subsequently processing of scanned data was done in software GOM ATOS, resp. GOM Inspect Professional.

\section{CT scanner}

As the second system, there was used CT scanner METROTOM $15002^{\text {nd }}$ generation $(225 \mathrm{kV} / 500$ W/MF) with operating system METROTOM OS 3.4.2.18129. Generally, in this case are used X-ray beams, which are generated in the X-ray tube. The radiation then emerges in the form of conical beam via the diaphragm towards the X-ray detector. First, the beams hit the workplace and then the detector surface. Depending on the geometry and the absorption characteristics of the workpiece, the X-ray beams are absorbed with different degrees of attenuation [6]. So finally $2 \mathrm{D}$ grey images are created in the detector. The workpiece is rotated step-by-step $360^{\circ}$ about the rotating axis, thus creating a large number of workpiece images. 3D image is then created during reconstruction, where are acquired data converted into a voxel mode. The setting of basic scanning parameters is given in Tab. 1. Voxel size in this case was equal to $121 \mu \mathrm{m}$.
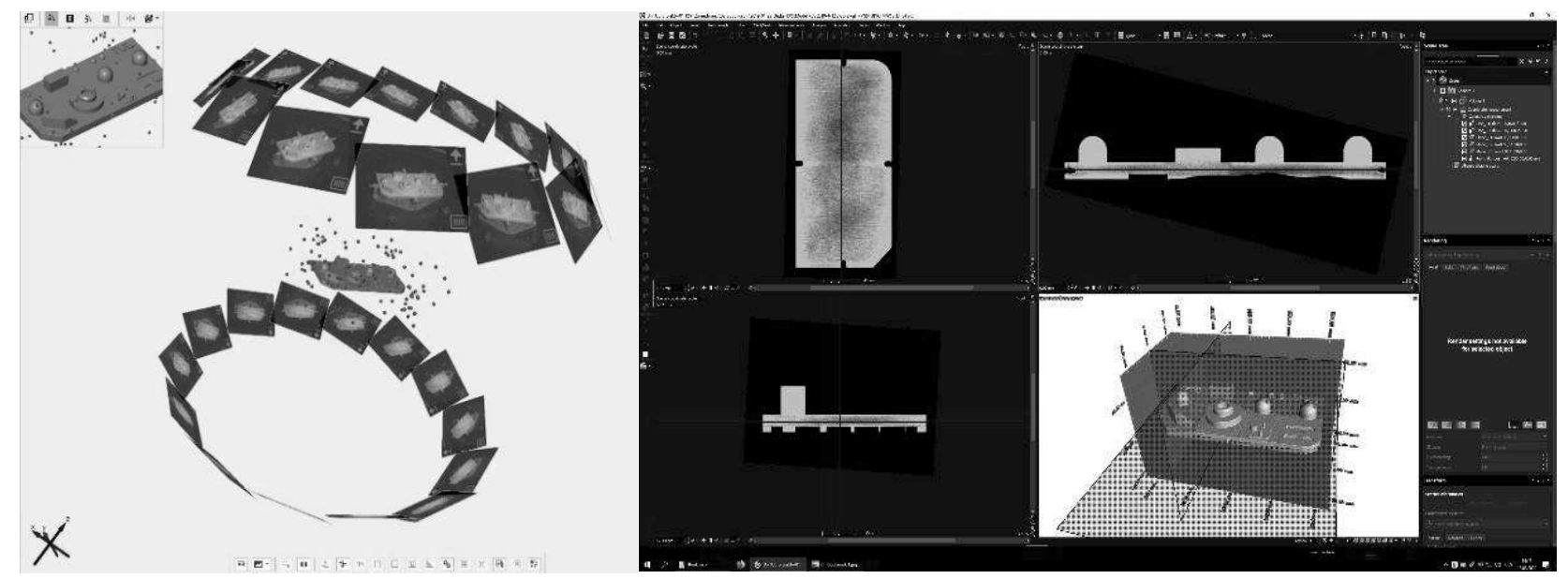

Fig. 1 Acquired scans at scanning by system ATOS (left) and working environment of VGSTUDIO MAX 3.2 (right) 
Tab. 1CT scanner METROTOM 1500 -basic scanning parameters

\begin{tabular}{|c|c|c|c|c|c|c|}
\hline $\begin{array}{c}\text { Acquisition } \\
\text { software }\end{array}$ & $\begin{array}{c}\text { Tube } \\
\text { voltage }\end{array}$ & $\begin{array}{c}\text { Tube } \\
\text { current }\end{array}$ & $\begin{array}{c}\text { Scan } \\
\text { method }\end{array}$ & $\begin{array}{c}\text { Integration } \\
\text { time }\end{array}$ & Filter & $\begin{array}{c}\text { Number of } \\
\text { projections }\end{array}$ \\
\hline $\begin{array}{c}\text { METROTOM OS } \\
\text { 3.4.2.18129 }\end{array}$ & $195 \mathrm{kV}$ & $1566 \mu \mathrm{A}$ & $\begin{array}{c}\text { VAST } \\
\text { (full_scan) }\end{array}$ & $667 \mathrm{~ms}$ & $\mathrm{Cu} 1.00 \mathrm{~mm}$ & 1300 \\
\hline
\end{tabular}

In Fig. 1 (right) is shown the working environment of software VGSTUDION MAX 3.2, which is divided into 4 windows ( 3 windows for 2D slices and last one for 3D data set).

\section{Model for scanning}

For the purposes of the experimental work, there was chosen specific model (product) - see Fig. 2. It serves as a special standard (can be termed also as "etalon"), designed for the analysis of dimensional accuracy and assessment of scannability of details. The model is made on a CNC machining centre from aluminium alloy. The standard was designed in such was to contain a large number of concave and convex ele-

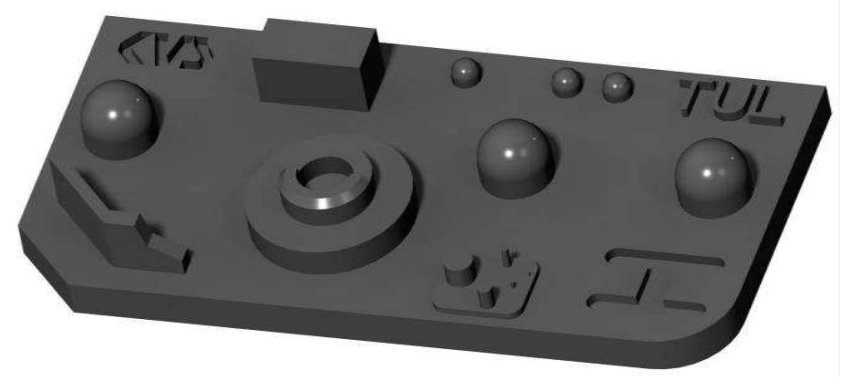

ments of various sizes. This makes it possible to evaluate the ability of scanners to digitize small geometric elements. Such standard also contains external and internal cylindrical elements and especially spherical elements, which are standardly used to assess the accuracy of scanning at certified tests. E.g. German company GOM verifies the accuracy of ATOS scanners by using the so-called Acceptance tests [7], which are carried out in accordance with the following standard VDI / VDE 2634 - Part 3 [VDI / VDE 2634 2008]. The standard (model) for the acceptance test also contains a set of spheres (one pair of spheres for each measuring volume) and for the basic evaluation are used diameters, different shapes and spacing (centre distances) of spheres $[8,9]$.

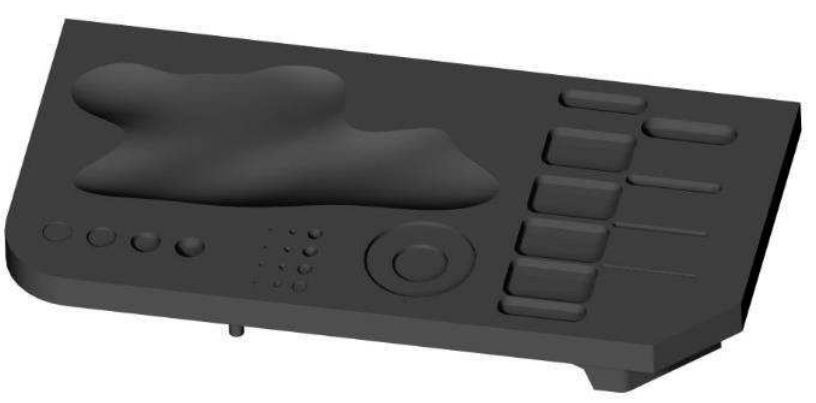

Fig. 2 Designed standard for evaluation scanning accuracy, (left - upper part, right - lower part)
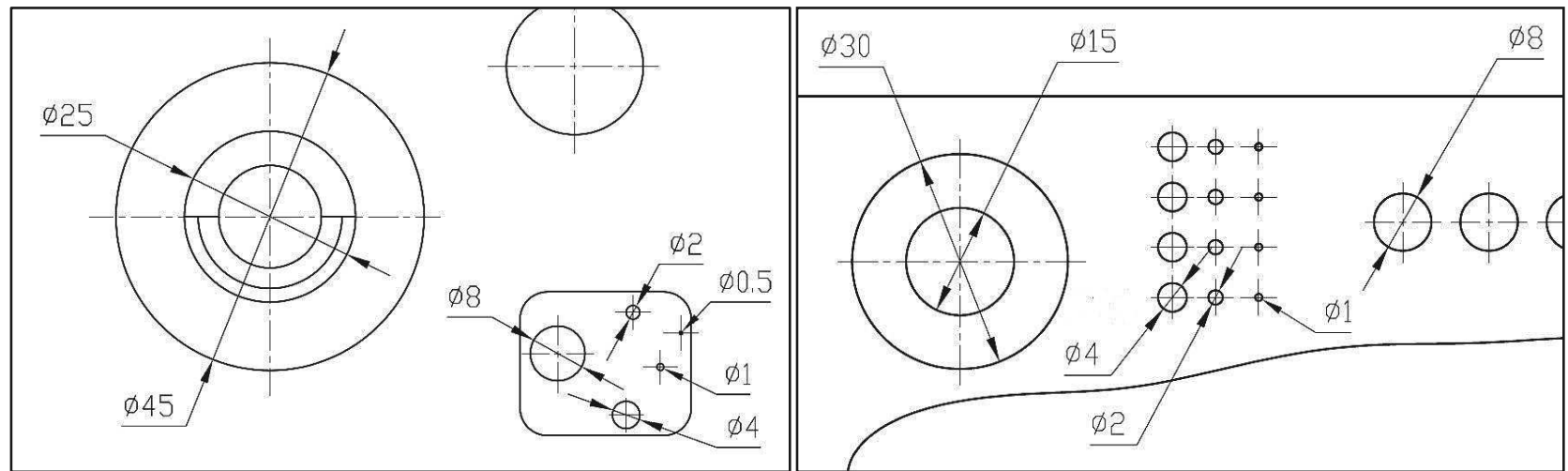

Fig. 3 Dimensions of the chosen elements on the scanned model, (left - cylindrical elements, right - holes)

The external dimensions of the used standard (model) are as following: $200 \times 100 \times 10 \mathrm{~mm}$. The set of larger spheres (designed for the application of measuring volume MV 320) is $\varnothing 20 \mathrm{~mm}$ and spacing between these spheres is subsequently 55,110 and 165 $\mathrm{mm}$. Moreover, cylindrical elements (see Fig. 3 - left) are divided into two basic groups. As the first group there is pair of larger coaxial cylinders (Ø 25 and 45 $\mathrm{mm})$, the second group is given by five cylinders of small diameters. These are created to determine the own limits of scanning devices. Such diameters are subsequently geometrically graduated (Ø $0.5,1,2,4$ and $8 \mathrm{~mm}$ ). The cylindrical hole systems, which are used for the analysis, are located at the bottom part of chosen standard and also have several graduated diameters (see Fig. 3 - right). The first system consists of two shallow cylindrical holes with a common axis and having larger dimensions (Ø 15 and $30 \mathrm{~mm}$ ). The remaining holes are divided into four sets, each set contains four holes of the same diameter $(\varnothing 0.5,1,2$, 
$4 \mathrm{~mm})$ and different depths $(0.5,1,2,4 \mathrm{~mm})$. Thus it is possible, among other quantities, to monitor the dependence of the scanned depth of hole on its diameter. These dependences are especially important for the $3 \mathrm{D}$ optical scanning scanners, because there is quite strong limitation for these devices about scanning internal geometry of the product. On the other hand, there isn't problem to scan these parts of the product in the case of CT scanner.

\section{Evaluation procedure}

The scanned models were verified mainly from the dimensional accuracy point of view by their comparison with the reference data measured from CMM measurements (these are subsequently marked as reference values) and quality of scanned data (ability of scanners to capture quite complex shapes, internal geometry, details of given elements, etc.). In addition to that, there were also computed so-called colour maps of deviations, which can easily visualize the real normal deviation of scanned models from each other in $3 \mathrm{D}$ space. It is thus possible to comprehensively compare a real dimensions of product with a nominal CAD model data (see Fig. 9 and Fig. 10) or e.g. compare two scanned models against each other.

Standardized procedures in software GOM Inspect were chosen for determination the dimensional characteristics (diameters, length dimensions, GD\&T). With respect to reality that own model created by 3D scanning is basically just a cloud of points (or more precisely polygonal mesh), the method of fitting elements by the Gaussian best-fit for $3 \sigma$ was chosen to define individual elements (see Fig. 4). From these data there were subsequently computed the arithmetic mean and measurement uncertainty for each monitored dimension. As the major subject of the scanning accuracy analysis, there was taken the difference between this mean value and the one measured from the CMM measurement.
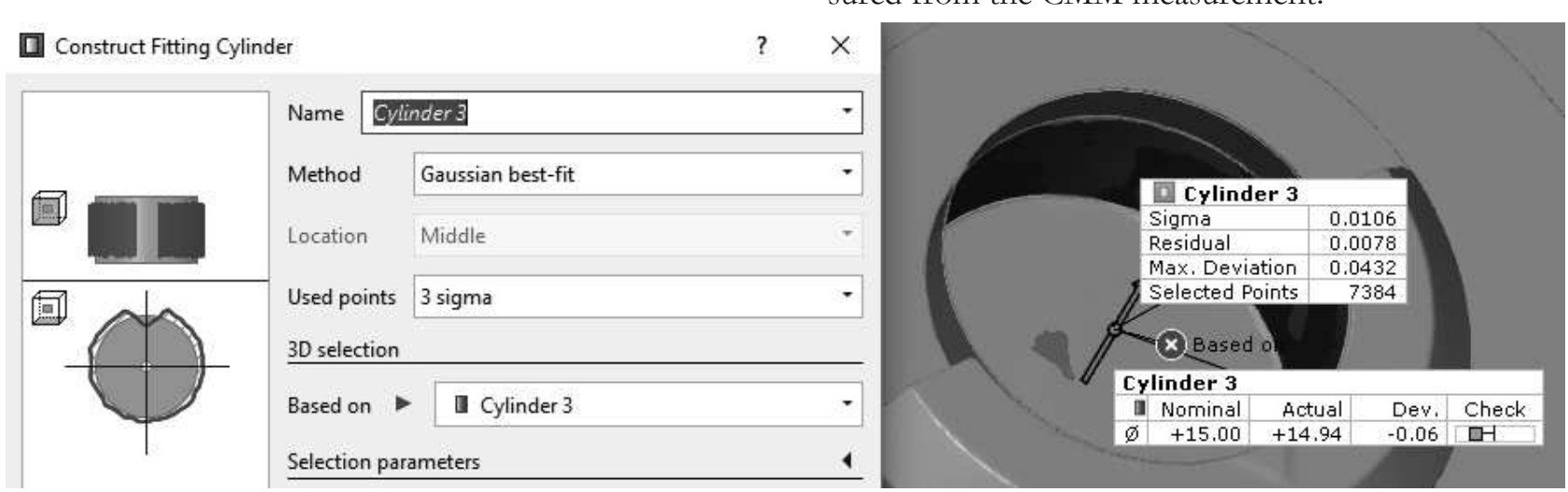

Fig. 4 Construction of the inner yylinder using Fitting Cylinder method

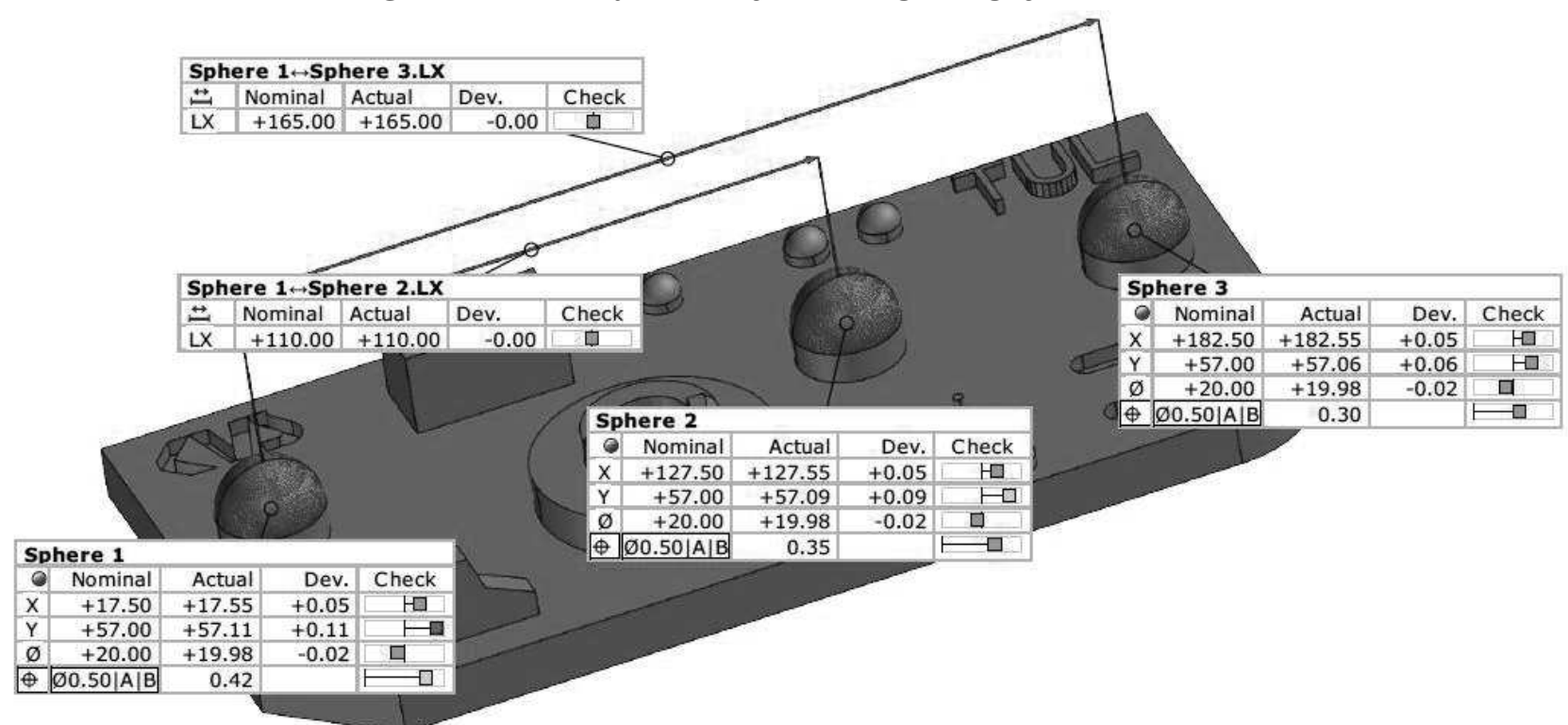

Fig. 5 Evaluation of the spherical elements via software GOM Inspect (Al model, METROTOM 1500)

An example how there were evaluated diameters of spheres and their mutual spacing for one of performed measurements is shown in the following Fig. 5, where are numerically and graphically illustrated differences between CAD data and results of CT scanner. In the two upper textboxes are given results for the monitored spacing between spheres - in this case for spacing as $110 \mathrm{~mm}$ (Sphere 1 - Sphere 2.LX) and also for 165 
$\mathrm{mm}$ (Sphere 1 -Sphere 3.LX). In this case are nominal values (CAD data) the same as actual values (measured data). However, they differ compared to CMM data. In addition to that, in the lower 3 textboxes are given diameters for that three monitored spheres. Nominal value in this case is $\varnothing 20 \mathrm{~mm}$, deviation is the same for all three monitored spheres and it is $-0,02 \mathrm{~mm}$. Another evaluation of the dimensional characteristic is given in chapter 6.

\section{Evaluation of dimensional characteristics}

As there was already written before, the chosen model (standard) was used to evaluate the measurement accuracy of both compared systems, in which the reference dimensions were determined by utilization high accuracy CMM device DEA GLOBAL Status 7.10.5 from company Hexagon Metrology Ltd. There were monitored three groups of dimensional characteristics - diameters and spacing of spherical elements, external cylindrical elements (cylinders) and internal cylindrical elements (holes). Individual measurements were performed according to the evaluation procedure summarized in the previous chapter 5. In Tab. 2 is given an example of dimensional characteristics results - in this case it is for the group of external cylindrical elements having diameters as following: 1 , 2, 4, 8, 25 and $45 \mathrm{~mm}$.

Tab. 2 Dimensions of the evaluated elements - outer cylindrical elements (Ø 1, 2, 4, 8, 25 and $45 \mathrm{~mm})$

\begin{tabular}{|l|c|c|c|c|c|c|}
\hline \multicolumn{2}{|c|}{ Given element } & \multicolumn{3}{c|}{ Measured dimensions } & \multicolumn{2}{c|}{ Deviations } \\
Notation & $\begin{array}{c}\text { Nominal } \\
\text { dimension } \\
{[\mathrm{mm}]}\end{array}$ & $\begin{array}{c}\text { CMM } \\
(\text { reference }) \\
{[\mathrm{mm}]}\end{array}$ & ATOS III & CT & ATOS III & CT \\
\hline Cylinder 1 & 1 & 0.975 & 1.01 & 0.95 & 0.035 & -0.025 \\
\hline Cylinder 2 & 2 & 1.980 & 2.00 & 1.96 & 0.020 & -0.020 \\
\hline Cylinder 4 & 4 & 3.976 & 4.01 & 3.96 & 0.034 & -0.016 \\
\hline Cylinder 8 & 8 & 7.976 & 8.01 & 7.97 & $\mathbf{0 . 0 3 4}$ & $-\mathbf{0 . 0 0 6}$ \\
\hline Cylinder 25 & 25 & 24.999 & 25.02 & 25.00 & $\mathbf{0 . 0 2 1}$ & $\mathbf{0 . 0 0 1}$ \\
\hline Cylinder 45 & 45 & 45.003 & 45.02 & 45.00 & $\mathbf{0 . 0 1 7}$ & $-\mathbf{0 . 0 0 3}$ \\
\hline
\end{tabular}

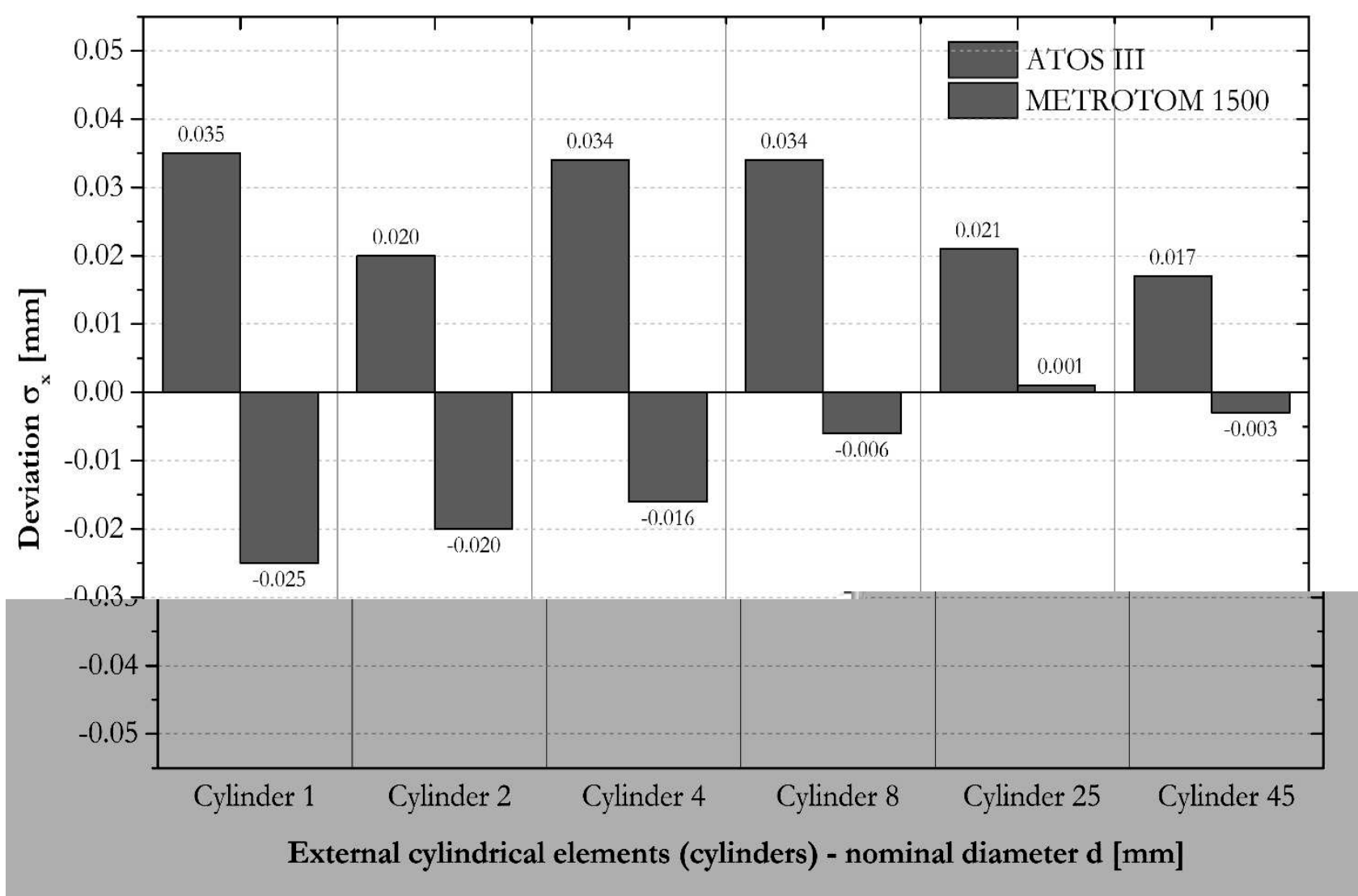

Fig. 6 Deviations of diameters for external cylindrical elements 
In Fig. 6 are graphically shown results, which are summarized in Tab. 2. For chosen external cylindrical elements are there given deviations both for system ATOS III and METROTOM 1500.
In Fig. 7 is shown the same type of graph as before, only in this case are summarized results for the internal cylindrical elements ( $\varnothing 1,2,4,8,15$ and $30 \mathrm{~mm})$.

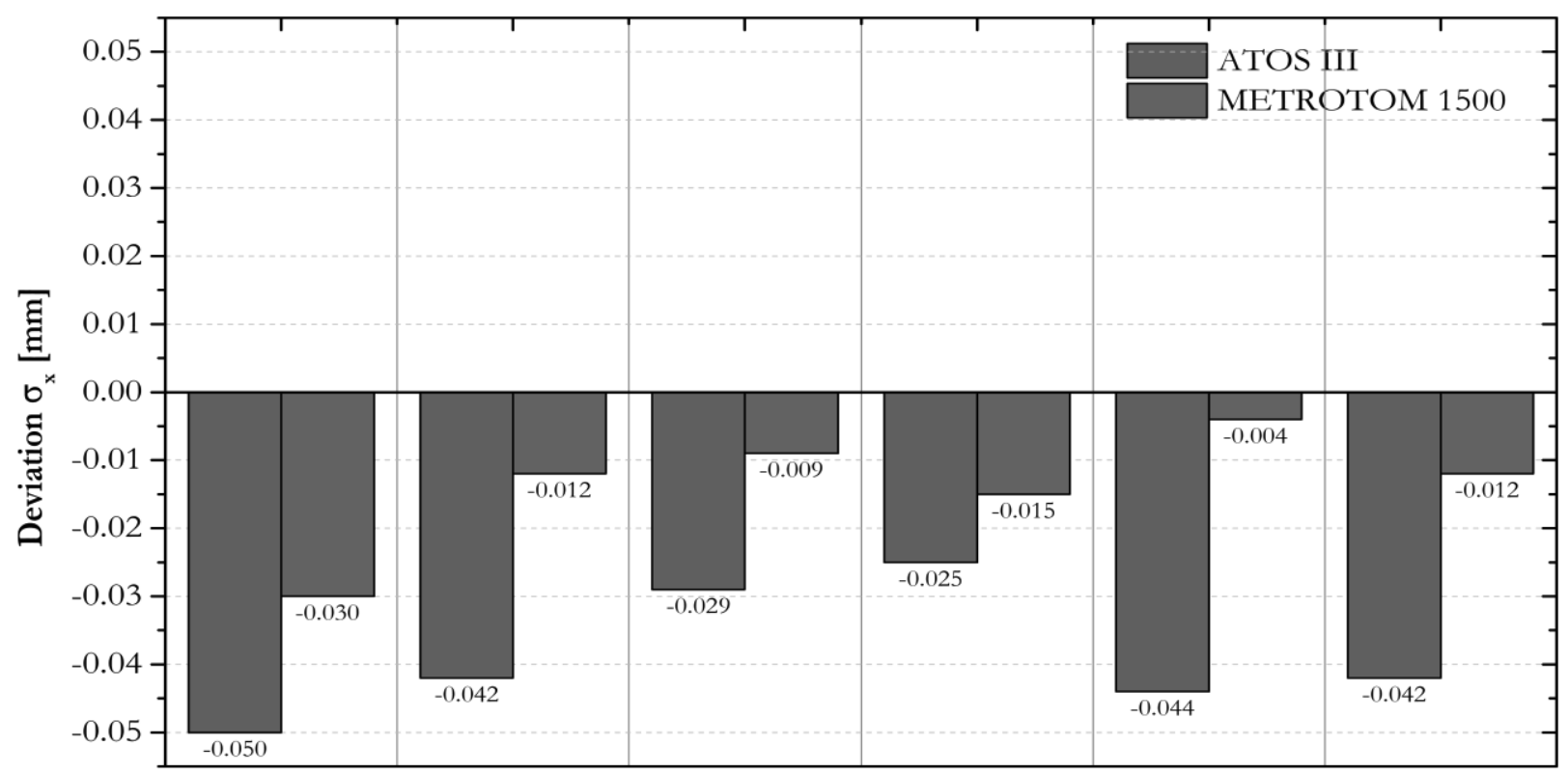

Hole 1

Hole 2

Hole 4

Hole 8

Hole 15

Hole 30

Internal cylindrical elements (holes) - nominal diameter $\mathbf{d}$ [mm]

Fig. 7 Deviations of diameters for internal cylindrical elements

Final graphical illustration of dimensional characteristics is given in Fig. 8 that shows deviations of different spacing between spheres of $\varnothing 20 \mathrm{~mm}$ as

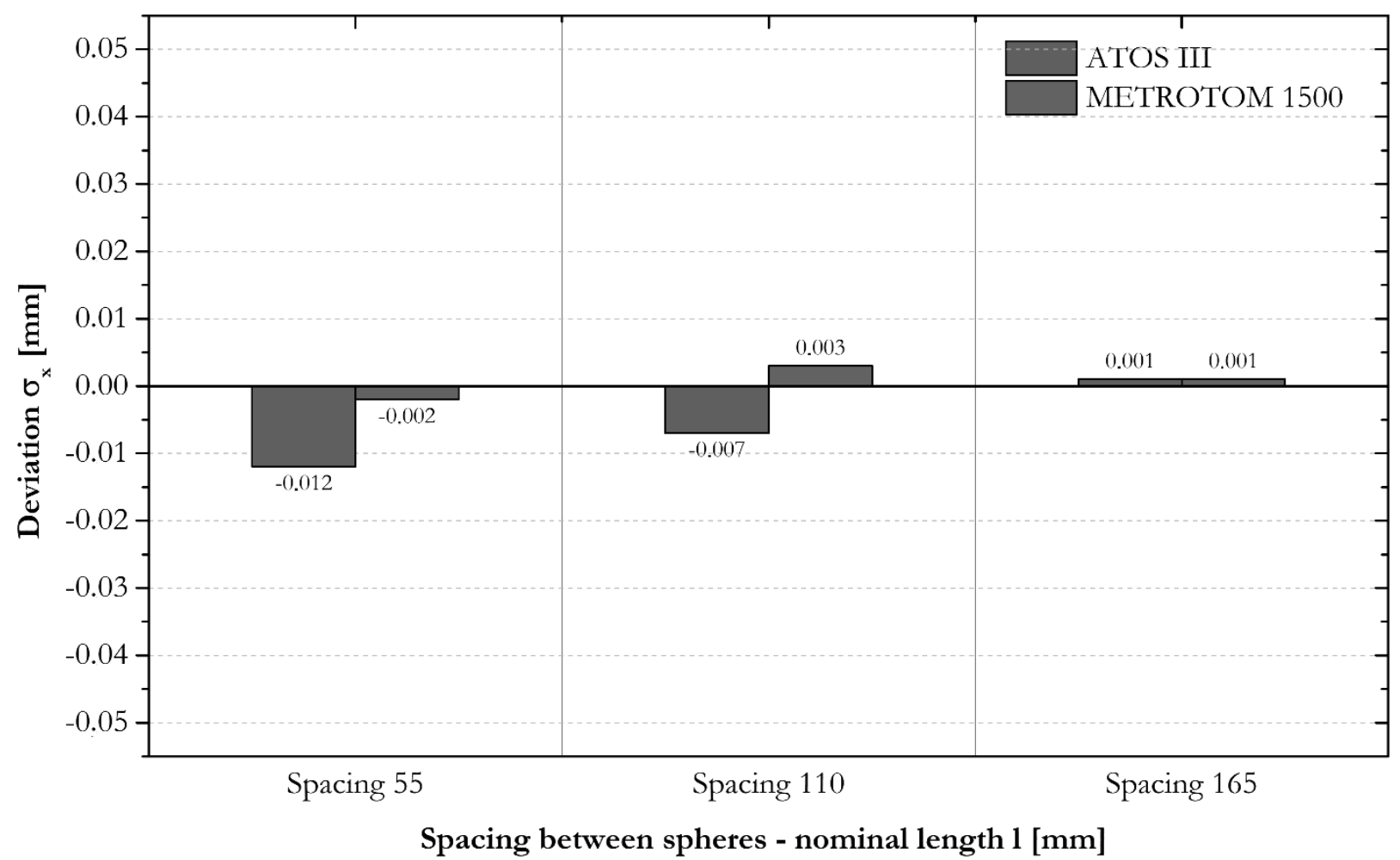

Fig. 8 Deviations of spacing between spheres of $\varnothing 20 \mathrm{~mm}$ 
Colour map of deviations between CAD data and data scanned by the optical 3D scanner ATOS III are shown in Fig. 9. Except the regions containing letters
KVS and TUL, there can be observed quite good similarity between given set of data both on the upper surface and on the edges of workpiece.

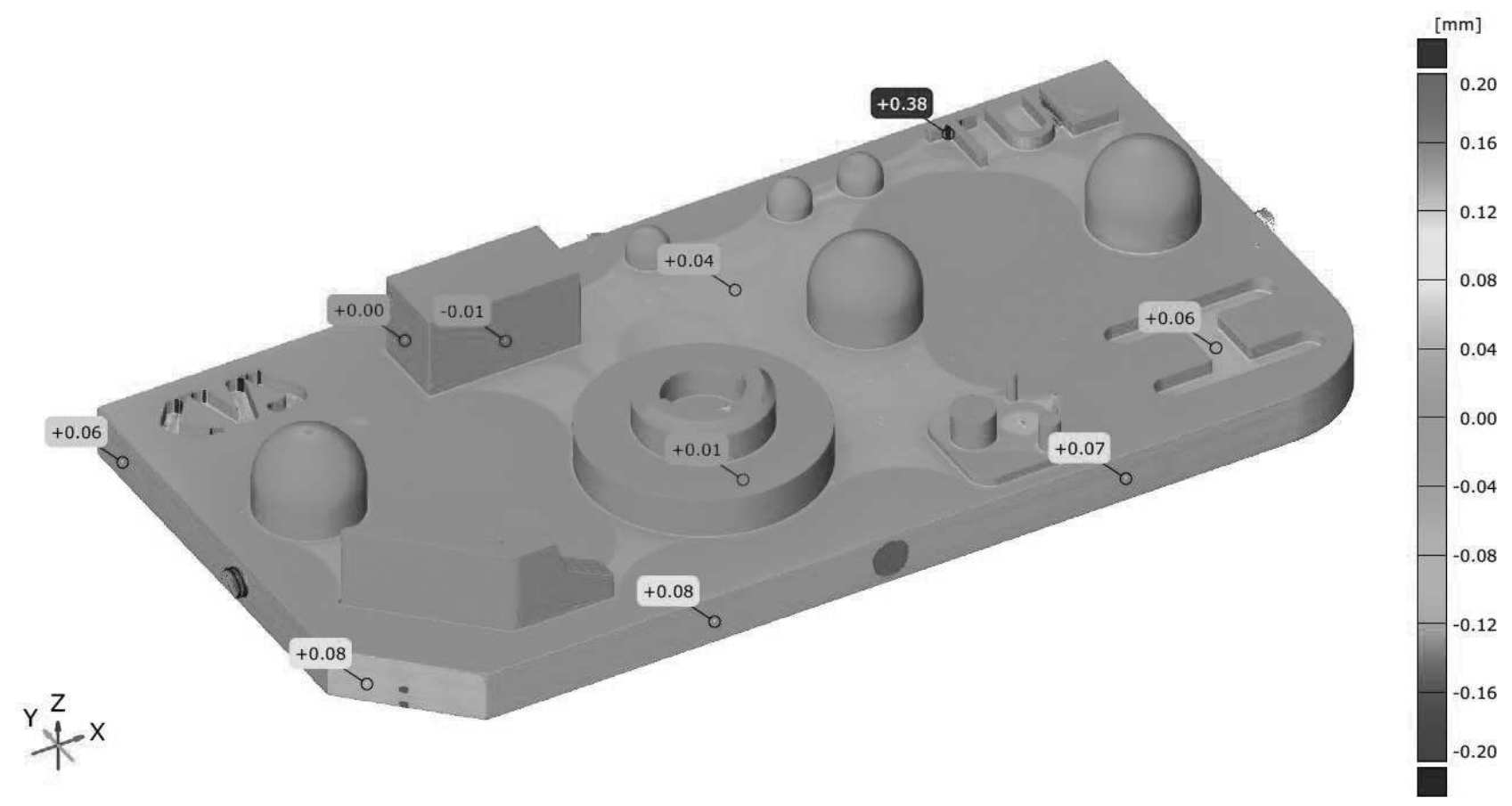

Fig. 9 Colour map of deviations (ATOS III vs. CAD)

In Fig. 10 is shown another colour map of deviations - in this case it is deviation between CAD data and data scanned from the CT scanner METROTOM $15002^{\text {nd }}$ generation. It is evident that vast majority of the scanned surface reveals the similar deviations as can be seen in Fig. 9. This is also valid for the edges of the workpiece, where are deviations almost identical. Similar deviations, which were shown in Fig. 9, can be found in the regions containing letters KVS and TUL. On the other hand, there can be found regions of rectangular shape, which reveal a wave-type shape and aren't observed neither on the real model nor in Fig. 9. It is obvious that these image errors (sometimes called as artifacts) are caused by the noise and standard ring artifacts. There will be more detailed about these image errors in chapter 8.

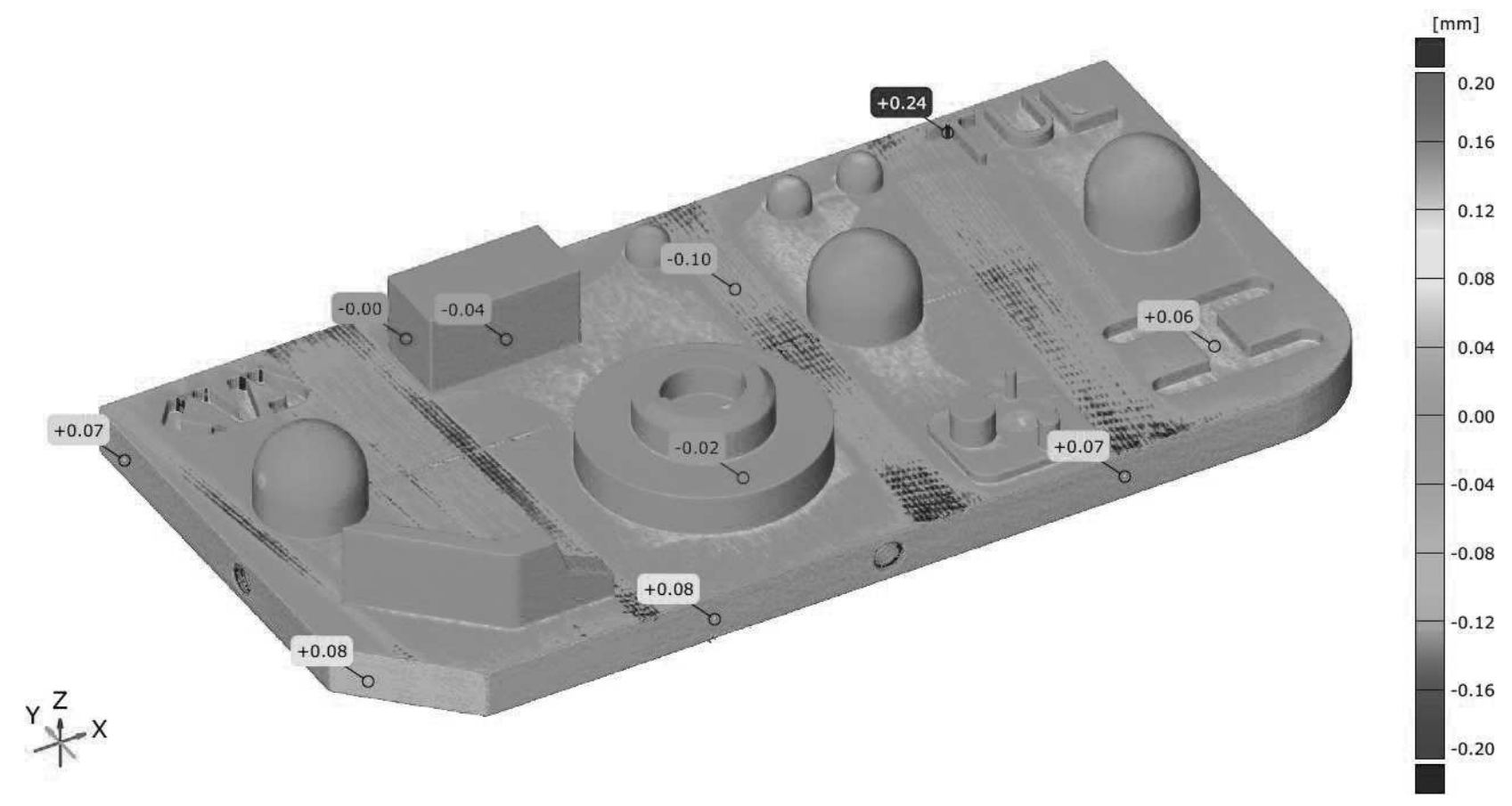

Fig. 2 Colour map of deviations (METROTOM 1500 vs. CAD 


\section{Evaluation of polygon meshes quality}

As the second monitored aspect, there was evaluated quality of the model created by 3D digitization. The overall quality of the polygon mesh (resolution, noise) and especially the ability of scanners to capture detailed areas (small holes, radii, ribs, etc.) or other problematic geometries, were monitored. Some selected points of interest are shown in the following figures, where is always polygon mesh from the ATOS III TripleScan optical scanner shown on the left and from the CT scanner METROTOM 1500 is shown on the right.

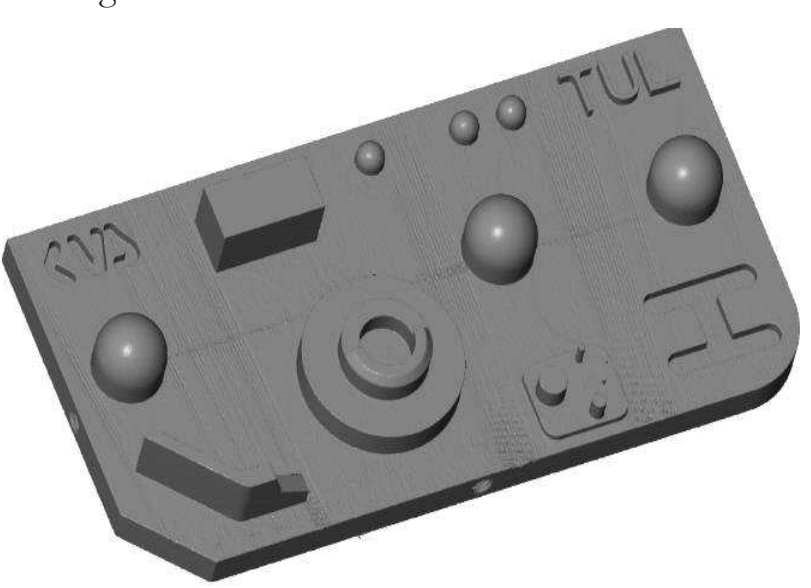

Fig. 11 Polygon mesh quality of the scanned standard (ATOS III - left, METROTOM 1500 - right)

In Fig. 12 is shown detail of the smaller cylinders (Ø 0,5, 1,4 and $8 \mathrm{~mm}$ ) both from the system ATOS

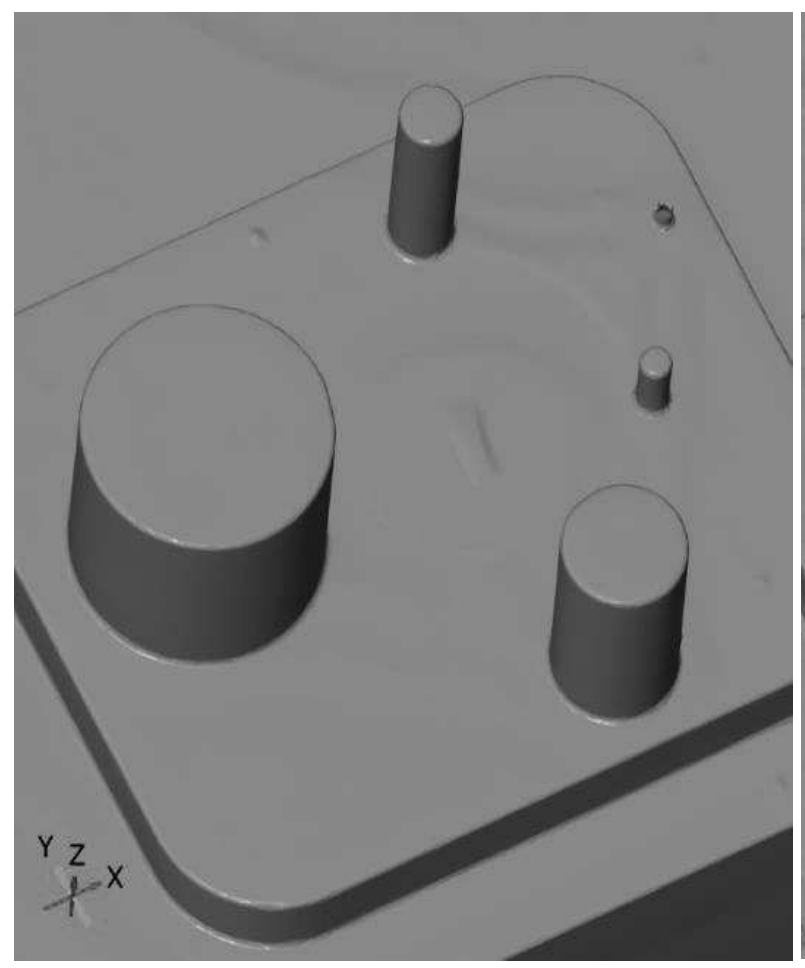

III and CT scanner METROTOM 1500.

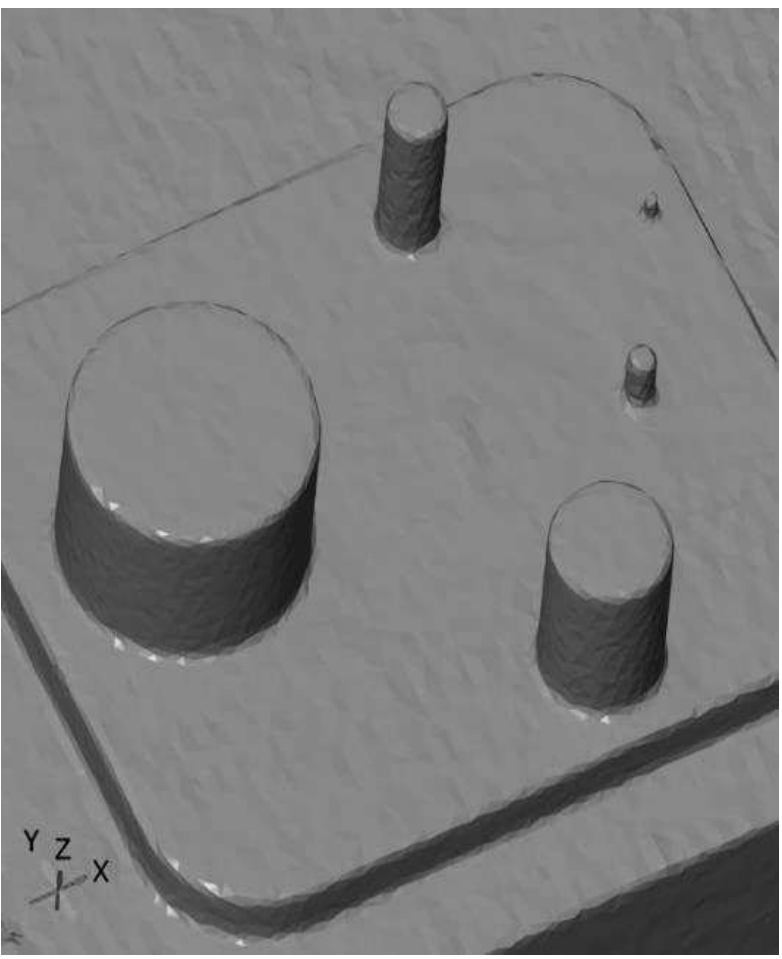

Fig. 12 Polygon mesh quality - detail of smaller cylinders (ATOS III - left, METROTOM 1500 G2 - right)

In Fig. 13 is shown another detail (now of holes) to indicate difference of the polygon mesh quality between system ATOS III and CT scanner METROTOM 1500. 

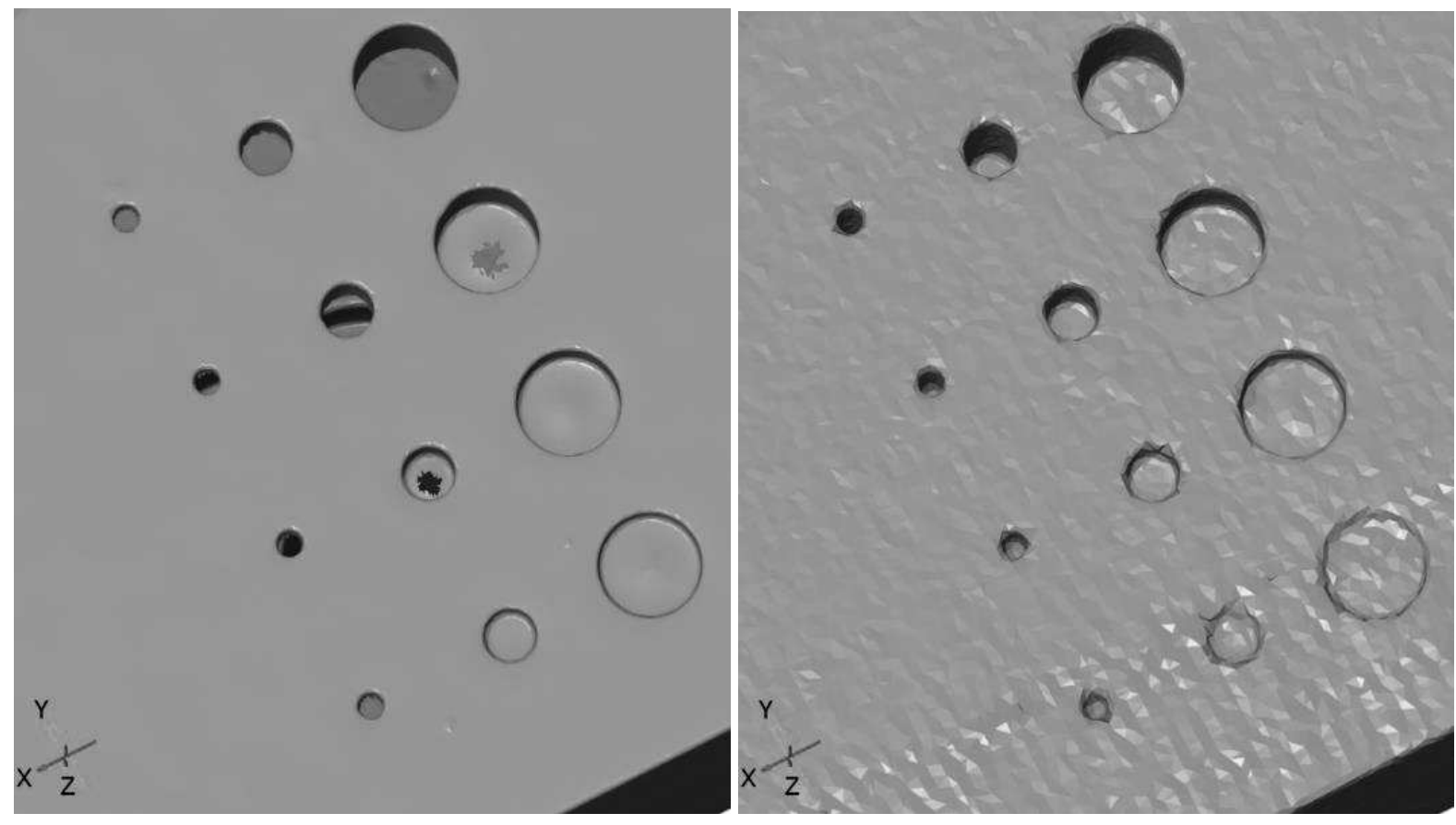

Fig. 13 Polygon mesh quality - detail of holes (ATOS III - left, METROTOM 1500 - right)

\section{Discussion of results}

From the accuracy analysis results about digitization from both systems (optical 3D scanner and CT scanner) are arising the following basic findings:

- The highest accuracy is achieved with such dimensions that do not depend on the quality of the scanned surface - typically at the centre distances between elements. As it follows from Fig. 8., error of the spacing between spheres ranged up to $12 \mu \mathrm{m}$ for the system ATOS III and only up to $3 \mu \mathrm{m}$ for the CT scanner METROTOM 1500.

- The dimensions measured by the ATOS III optical scanner were larger for external elements (see Fig. 6) and smaller for the internal elements (see Fig. 7) compared to the reference values. The deviation of diameter reached on average around $30 \mu \mathrm{m}$. Slightly worse results were measured with the holes. This corresponds to the expected thickness of antireflection coating that had to be used before the own optical scanning. Assuming that the spray coating thickness is around $0.015 \mathrm{~mm}$, the diameter of the external element will be by $0,03 \mathrm{~mm}$ larger and on the other hand, size of the internal element will be smaller by this value. Thus to increase the accuracy, it is therefore advisable to use coatings with the smallest possible thickness (e.g. titanium coating $\mathrm{TiO}_{2}$ ). It can be also observed from the results that the magnitude of error usually doesn't depend on the nominal size of given element. Only in the case of elements, which do not have enough area for scanning - for example holes having small diameters (in Fig. 7 it is valid for holes with a nominal diameter of 1 and $2 \mathrm{~mm}$ ) or holes with small depth (in Fig. 7 it is valid for the holes with a nominal diameter of 15 and $30 \mathrm{~mm}$ that have a height of only $1.5 \mathrm{~mm}$ ), thus there may be a slight increase of error in these cases.

- Deviations detected by the computed tomography reached negative values both for external elements (see Fig. 6) and for internal elements (see Fig. 7) dimensions. The error varied from $-30 \mu \mathrm{m}$ up to zero deviations. Especially from the graph for the external cylindrical elements (see Fig. 6) is obvious a certain trend of error decreasing with increasing nominal size of the element. While cylinders with a nominal diameter $1-4 \mathrm{~mm}$ showed an error of diameter around $-20 \mu \mathrm{m}$, cylinders with a nominal diameter over $8 \mathrm{~mm}$ achieved errors of max. $6 \mu \mathrm{m}$.

From the quality of resulting polygon mesh point of view and the ability of the individual scanners to 
capture problematic areas can be concluded following major findings and conclusions:

- The undeniable advantage of the computed tomography rests in the ability to scan also the internal structures and internal geometry of the product (see Fig. 12 and Fig. 13), where are evident unscanned regions (green colour) in the polygon mesh in the pictures on the left, which belongs to the ATOS III system. If it is necessary to digitize optically inaccessible regions, computed tomography is almost the only available non-destructive solution. On the other hand, there are limitations due to the working space of the machine and in some cases by the shape and material of the workpiece. This is also confirmed by the chosen model for scanning, where can be in Fig. 10 and also in Fig. 11 (right) observed image errors (sometimes called as artifacts). There is a relatively large number of different image errors as e.g. blurring (double edge), ghost images (double images), noise, beam hardening, standard ring artifacts or insufficient projections. In this case it is noise on the upper and lower part of the model. Moreover, there were observed standard ring artifacts on the lower part of the model. Noise in the computed tomography is caused due to random variation of the nominal grey scale values both in the air and in the material. If the variation is too high, air and material are perceived as more or less noisy. As a solution there e.g. should be increase integration time, number of projections or (and) image averaging. Generally, everything that cause more photons to be used per generated voxel. Standard ring artifacts are caused by the different sensitivity of the detector pixels due to the different distance from the rotary axis [6].

- The resolution of the resulting model polygon mesh is in a way dependent on the size of scanned workpiece. In the case of the system ATOS III it is determined by the used measuring volume and resolution of the CCD chip of cameras. In the case of the CT scanner, it is basically done by the material and thickness of the scanned workpiece, its distance from the X-ray tube and resolution of detector. From these basic quantities are subsequently adjusted other parameters as e.g. tube voltage and current, integration time and so on. In light of resolution, size of voxel can be taken as the most important one. Although the theoretical resolution of both systems was almost identical (ATOS III - $100 \mu \mathrm{m}$ and METROTOM $1500-121 \mu \mathrm{m}$ ), the results show that the real resolution and polygon mesh quality of the system ATOS III is significantly better - e.g. it contains lower noise (see Fig. 12 and Fig. 13) From these figures can be observed also more captured details in the case of the optical scanner ATOS III and can be also observed that lower resolution of CT scanner leads to the suppresses of details in the polygon mesh.

\section{Conclusion}

The major aim of this paper was to compare two scanners (optical 3D scanner ATOS Triple Scan and CT scanner METROTOM 1500) in light of their dimensional accuracy and polygonal mesh quality. In addition to that there was also effort to highlight advantages and disadvantages of these systems. As a model (standard) for their mutual comparison was chosen product, which was primarily designed to evaluate dimensional accuracy and scannability of details. This aluminium alloys model is made on a $\mathrm{CNC}$ machining centre and contains a large number of concave and convex elements. After scanning were measured data (resp. cloud of points) transferred into evaluation software GOM Inspect. Subsequently were these data compared with the reference ones (measured from CMM) and between themselves. With the help of detailed view, there was also evaluated the polygon mesh quality for both used scanners.

In conclusion, there can be stated that the performed analysis confirmed the assumption about ability of the computed tomography system to easily scan the internal geometry of the workpiece. On the other hand, it was seen that the optical scanner that is working on the principle of Fringe Projection and active triangulation, allows to achieve a better quality polygon mesh with more details. In this case performance of CT scanner strongly depends on the workpiece material and thickness and its distance from the $\mathrm{X}$-ray tube. With respect to this reality, these advantages of optical 3D scanner will be apparent for reverse engineering, where polygon mesh quality is very important. System ATOS III or other systems working on the similar principles, will be also a better solution at digitalizing large products. 
Regarding the dimensional accuracy, both systems provide very good results and individual errors correspond to the manufacturer's data. Within the frame of this experiment, better results (errors up to about 10 $\mu \mathrm{m})$ were achieved by the computed tomography system. Slightly worse results were measured in the case of optical 3D scanner ATOS III - errors of lengths and diameters varied around $30 \mu \mathrm{m}$. Nevertheless, this was caused by applying an anti-reflection spray on the measured object.

The preparation and adjustment of product and devices to carry out digitization took about 15 minutes in the case of the ATOS scanner (application of reference points and anti-reflection spraying, fixing the model on the measuring table), own scanning took 15 minutes and subsequent post-processing (merging of two measuring series, calculation of mesh) took 10 $\mathrm{min}$. So totally after $40 \mathrm{~min}$ there was at disposal a polygon model available, ready for inspection or reverse engineering. On the other hand, it took about $10 \mathrm{~min}$ to prepare workpiece for scanning in the case of CT scanner - just to fix the model in the polystyrene jig and put it on the rotary table. Own scanning strongly depends on the size and material of the workpiece, as well as on the scanning parameters (mostly scanning time and image averaging) - in this case it took $50 \mathrm{~min}$ together with basic post-processing (surface determination and registration). Thus from the duration point of view can be taken these systems as similarly. Last but not least, there should be mentioned initial cost that in about four time higher in the case of CT scanner. Nevertheless, computed tomography is becoming increasingly accurate and less expensive.

\section{Acknowledgement}

This paper was supported by the institutional support of the Technical University of Liberec under the project No. 117/2200 and No. 117/2400.

\section{References}

[1] SEDLAK, J. et al. (2017). Shape Inspection of Gear Prototypes Using Reverse Engineering Method. Manufacturing Technology [online]. 2017, 17(6), pp. 945-952. ISSN 12132489, 12132489. Available at: doi:10.21062/ujep/x.2017/a/12132489/MT/17/6/945
[2] VÁRADY, T., MARTIN, R.R. and COX, J. (1997). Reverse engineering of geometric models - An introduction. CAD Computer Aided Design [online]. 1997, 29(4), pp. 255-268. Available at: doi:10.1016/s0010-4485(96)00054-1

[3] BARBERO, B.R. and URETA, E.S. (2011). Comparative study of different digitization techniques and their accuracy. Computer-Aided Design [online]. 2011, 43(2), 188-206. ISSN 0010-4485. Available at: doi:10.1016/j.cad.2010.11.005

[4] MENDŘICKÝ, R. (2018). Impact of Applied Anti-Reflective Material on Accuracy of Optical 3D Digitisation. In: Materials Science Forum [online]. Switzerland: Trans Tech Publications, 2018, pp. 335-344. Novel Trends in Production Devices and Systems IV. ISBN 978-30357-1265-0.

Available at: doi:10.4028/www.scienti-

fic.net/MSF.919.335

[5] PALOUSEK, D. et al. (2015). Effect of matte coating on 3D optical measurement accuracy. Optical Materials [online]. 2015, 40, pp. 1-9. ISSN 0925-3467. Available at: doi:10.1016/j.optmat.2014.11.020

[6] ZEISS METROTOM - Carl Zeiss Industrielle Messtechnik - PDF Catalogs | Technical Documentation | Brochure. METROTOM OS Operating Instruction [online]. [vid. 2020-08-18]. Available at: https://pdf.directindustry.com/pdf/carl-zeiss-industrielle-messtechnik/zeiss-metrotom/210459-842999.html

[7] GOM, GmbH. GOM Acceptance Test - Process Description, Acceptance Test according to the Guideline VDI/VDE 2634 Part 3. B.m.: Braunschweig, Germany. 2014

[8] FRKAL, M. (2016). Návrh kalibračního etalonu pro stanovení přesnosti měření optických 3D skenerů. Liberec, 2016. Diploma thesis. Technical Univeristy of Liberec.

[9] MENDRICKY, R. (2016). Determination of Measurement Accuracy of Optical 3D Scanners. MM Science Journal [online]. 2016, 2016(06), pp. 1565-1572. ISSN 18031269, 18050476.

Available at: doi:10.17973/MMSJ.2016_12_2016183 